Somnologie 2010 $14: 5-5$

DOI 10.1007/s11818-010-0460-2

Online publiziert: 12. März 2010

๑) Springer-Verlag 2010

\author{
B. Högl \\ Bereich Schlafmedizin, Universitätsklinik für Neurologie, \\ Medizinische Universität Innsbruck
}

\title{
Aktuelle österreichische Schlafforschung
}

Die „Somnologie“ ist nicht nur das bewährte und hochgeschätzte Fachjournal der Deutschen Gesellschaft für Schlafmedizin (DGSM), sondern seit 2008 auch der Österreichischen Gesellschaft für Schlafmedizin und Schlafforschung (ÖGSM/ ASRA).

Daher freuen wir uns besonders, das Jahr 2010 mit einem Sonderheft zum Themenschwerpunkt Österreich beginnen zu dürfen. Die Beiträge in diesem Heft geben nicht nur einen Einblick in einige Themen der aktuellen österreichischen Schlafforschung, sondern sie befassen sich auch inhaltlich mit aktuellen Schlafgewohnheiten der Bewohner unserer Republik:

Die Arbeiten von Josef Zeitlhofer („Die Schlafgewohnheiten der Österreicher") und von Tina Falkenstetter („Erhöhte Tagesschläfrigkeit in Österreich") gehen zurück auf eine repräsentative Umfrage, welche von der Österreichischen Gesellschaft für Schlafmedizin und Schlafforschung in Auftrag gegeben wurde.

Die wichtigsten Ergebnisse dieser Umfrage sind im ersten Beitrag von Josef Zeitlhofer zusammengefasst. Ein interessanter Aspekt hierbei ist, dass sich die Schlafdauer der Österreicher reduziert hat, d. h. dass der Anteil der Kurzschläfer zugenommen und der Anteil der Langschläfer abgenommen hat.

\section{(7) Die Schlafdauer der Österreicher hat sich reduziert}

In der komplementären Arbeit über Tagesschläfrigkeit zeigt Tina Falkenstetter erstmals eine sehr hohe Monotonieintoleranz und erhöhte Einschlafneigung am Tage in der österreichischen Bevölke- rung. Hier konnte nicht nur ein Zusammenhang mit bekannten Risikofaktoren gefunden werden (anamnestische Hinweise auf schlafbezogene Atmungsstörungen usw.), sondern auch erstmals ein klarer inverser Zusammenhang mit der nächtlichen Schlafdauer: d. h. je kürzer die nächtliche Schlafdauer (Schlafmangelsyndrom), umso häufiger war Tagesschläfrigkeit.

Über eine aktuelle Umfrage zu Schlafgewohnheiten bei 330 Schulkindern berichtet Kerstin Hödlmoser. Die Studie weist auf den Zusammenhang mit Verhaltensauffälligkeiten hin. Gerhard Klösch stellt eine Studie der Österreichischen Bundesarbeitskammer bei 4200 Arbeitnehmern zum engen Zusammenhang zwischen Schlafstörungen und Arbeitsfähigkeit vor. Ein wichtiges Fazit dieser Arbeit ist, dass der öffentliche Diskurs über nichterholsamen Schlaf und den Zusammenhang mit der Arbeitswelt intensiviert werden sollte.

Abgesehen von geringfügigen regionalen Besonderheiten kann man mit Sicherheit davon ausgehen, dass Schlafprobleme und Schlafgewohnheiten der Bevölkerung in Österreich und in Deutschland große Übereinstimmung zeigen. Auch die gesundheitspolitischen Fragen, mit denen sich die Schlafmedizin auseinanderzusetzen hat, sind ähnlich.

Aus diesem Grund ist die enge $\mathrm{Zu}$ sammenarbeit der nationalen Schlafgesellschaften besonders wichtig - dies gilt natürlich in besonderem Maße für die $\mathrm{Zu}$ sammenarbeit zwischen den deutschsprachigen Gesellschaften DGSM und ÖGSM/ ASRA. Aufgrund der Interdisziplinarität der Schlafmedizin und der daraus entste- henden Herausforderungen für Qualitätssicherung in Ausbildung und Routine ist darüber hinaus eine enge Zusammenarbeit auf europäischer Ebene mit möglichst weitgehender Vereinheitlichung von Qualitätssicherungsmaßnahmen und Ausbildungsrichtlinien notwendig und teilweise auch schon etabliert. Die Akkreditierung von Laboren wird seit 1998 auch in Österreich routinemäßig durchgeführt.

\section{$\checkmark$ Auch in Österreich laufen die Vorbereitungen für eine Spezialisierung in Schlafmedizin}

Die DGSM hat mit der Einführung einer strukturierten Ausbildung zum Somnologen mit einer Prüfung zunächst vor der Fachgesellschaft, seit kurzem auf Zusatz-Facharztniveau vor den Ärztekammern hier europaweit sicherlich eine Vorreiterfunktion und die Latte sehr hoch gelegt. Auch in Österreich laufen derzeit die Vorbereitungen für eine Spezialisierung in Schlafmedizin. Ein Ausdruck der engen Zusammenarbeit zwischen DGSM und ÖGSM ist auch die vorliegende Ausgabe der Somnologie, die interessante Lektüre verspricht.

\section{Korrespondenzadresse Prof. Dr. B. Högl}

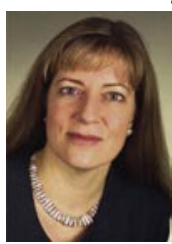

Bereich Schlafmedizin Univ.klinik f. Neurologie Med. Universität Innsbruck Anichstraße 35, 6020 Innsbruck Österreich birgit.ho@i-med.ac.at 